\title{
Perioperative Plasma Cortisol Levels during Transsphenoidal Operation of Pituitary Adenoma in ACTH Sufficiency and ACTH Deficiency
}

Henrik Borg' ${ }^{1}$ Sigridur Fjalldal', Peter Siesjöㄹ, Babar Kahlon², Eva Marie Erfurth'

1 Department of Endocrinology, 2 Department of Neurosurgery, Skåne University Hospital, Lund, Sweden

Fig 1. ACTH sufficient pts without cortisone substitution

700

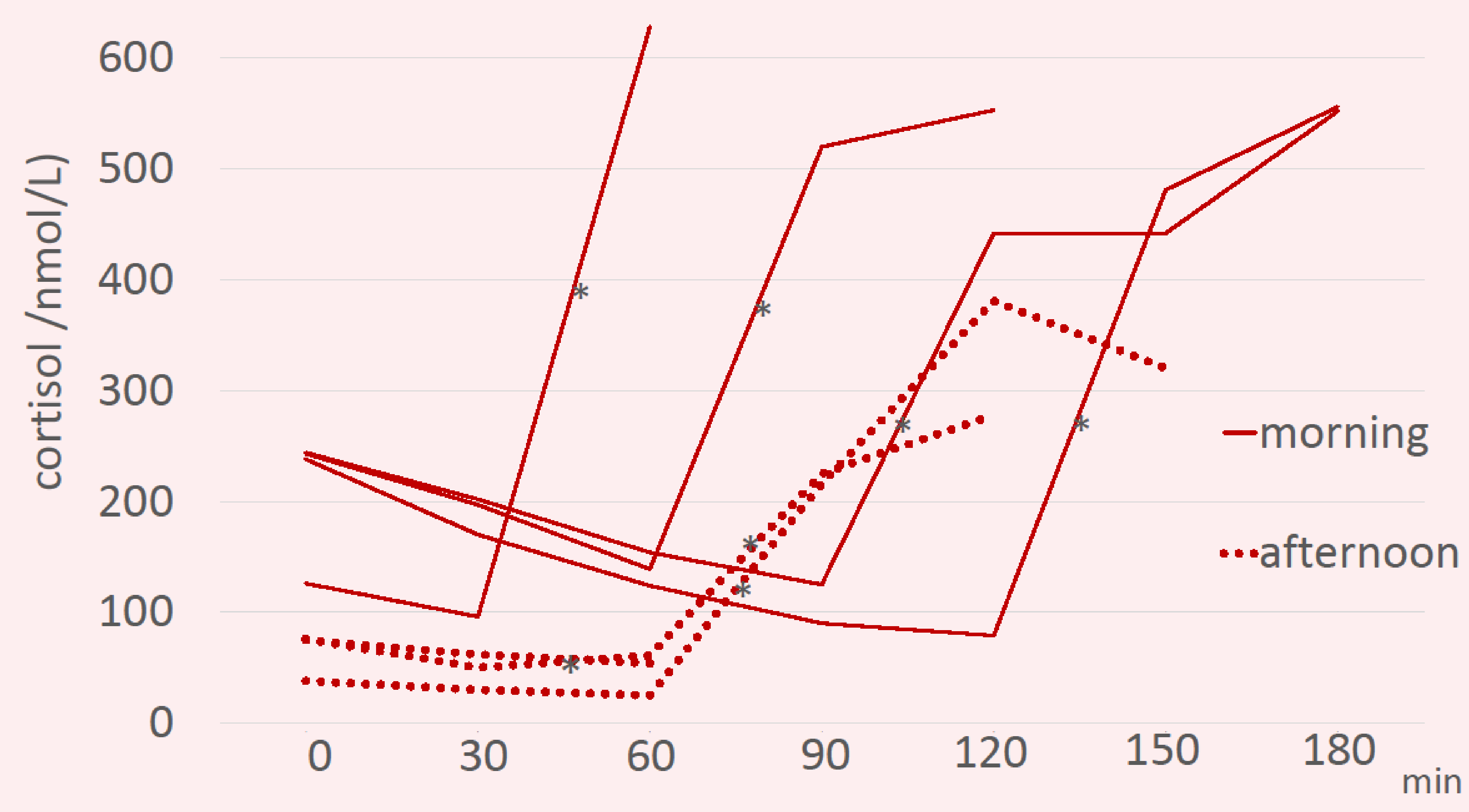

\section{Results I}

ACTH sufficient pts $(n=7)$ not receiving cortisone substitution (Fig. 1)

At the start of surgery, cortisol levels were $38-244 \mathrm{nmol} / \mathrm{L}$. Pts undergoing surgery in the morning $(n=4)$ had $126-244 \mathrm{nmol} / \mathrm{L}$, whereas those undergoing surgery in the afternoon $(n=3)$ all had lower levels, 38-76 $\mathrm{nmol} / \mathrm{L}$.

During the first part of the surgical - procedure, during entrance to the nasal cavity and paranasal sinuses, cortisol levels went down to $79-139$ and $24-54 \mathrm{nmol} / \mathrm{L}$ in the morning and afternoon groups, respectively.

After surgery had reached into the sella tursica $\left(^{*}\right)$ rising cortisol levels were noted in 6 of 7 pts; rising with $(\Delta) 217-532 \mathrm{nmol} / \mathrm{L}$ up to $278-628 \mathrm{nmol} / \mathrm{L}$.

In those who had surgery in the morning cortisol levels rose with $(\Delta) 402-532 \mathrm{nmol} / \mathrm{L}$. In 2 of the 3 pts who had surgery in the afternoon cortisol rose with 217 and 356 $\mathrm{nmol} / \mathrm{L}$, respectively, whereas in one patient no increase was observed.
Fig 2. Pts with hydrocortisone substitution

(ACTH sufficient and deficient)

--50 mg (sufficient)
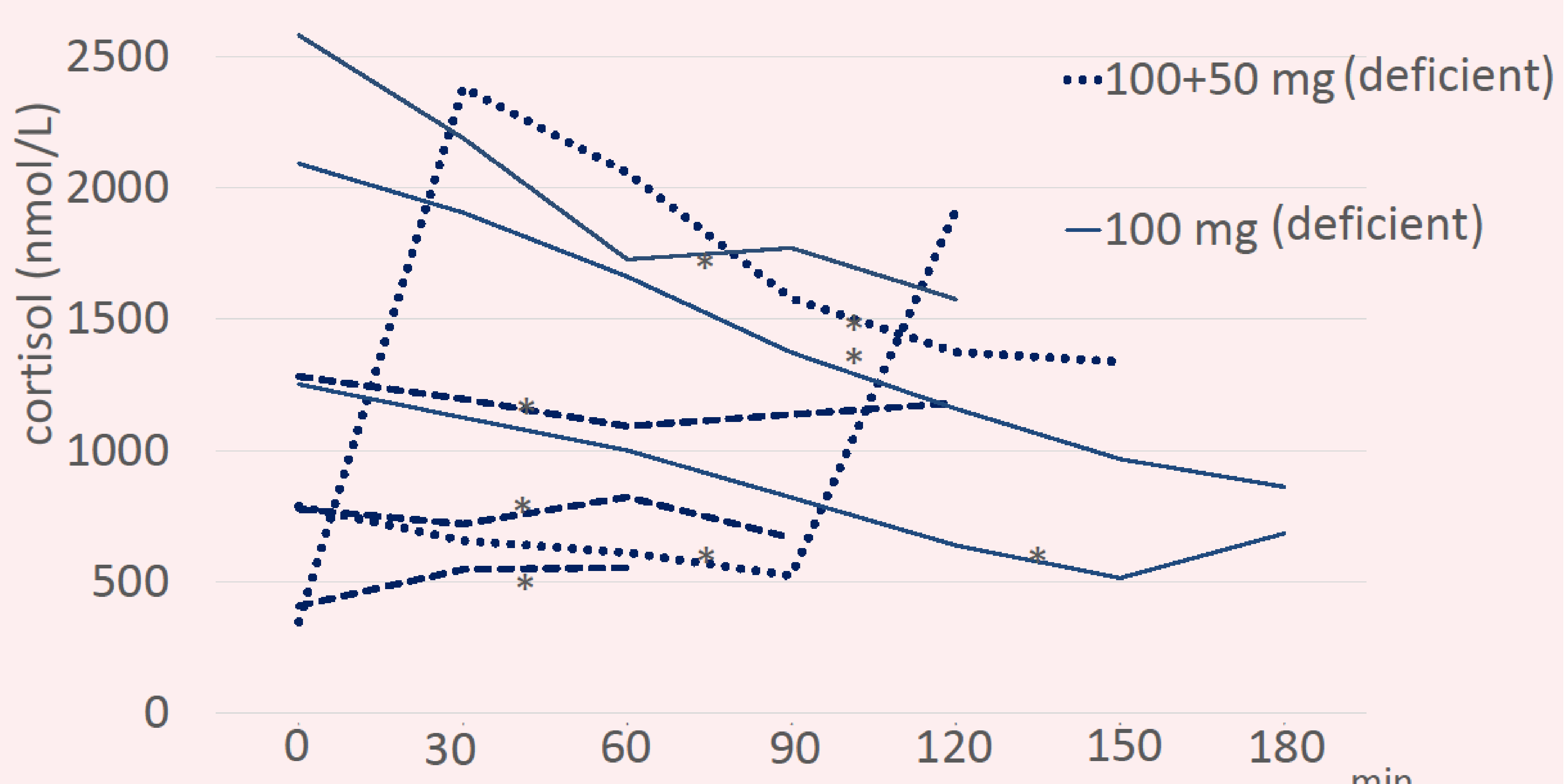

030

60

90

120

150

180

\section{Conclusions}

Cortisol levels increased after mechanical impact on the pituitary with normal ACTH function.

The highest increases were observed in pts without cortisone replacement who had morning surgery.

The low cortisol levels during the first part of surgery may partly be caused by the anesthetics used, propofol and remifentanil.

\section{Very high cortisol levels were achieved after our routine iv hydrocortisone substitution.}

\section{Postoperative cortisol function}

Cortisol levels were measured on day 3 after surgery; 8 of 10 pts retained normal adrenal reserve, whereas the remaining 2 were given oral hydrocortisone replacement therapy at discharge from the hospital.

\section{Results II}

\section{Introduction and objectives}

Our aim was to measure the serum cortisol secretion during endoscopic adenoma surgery in ACTH sufficient and deficient pts.

\section{Material and methods}

15 pts with surgery for pituitary adenoma were studied. Ages were 28-84 years. Serum cortisol was measured every 30 minutes from the start of surgery. The anesthetics used were propofol plus remifentanil.

$10 \mathrm{pts}$ had normal ACTH function, defined as a p-cortisol $>400 \mathrm{nmol} / \mathrm{L}$ in the morning or $>550 \mathrm{nmol} / \mathrm{L}$ after a $250 \mu \mathrm{g}$ ACTH stimulation test. Of the 10 pts 7 were not given any perioperative hydrocortisone $(\mathrm{HC})$ supplementation (Fig. 1), whereas 3 of 10 got the routine 50 mg intravenous HC at the start of anesthesia (Fig. 2). Five pts with known ACTH deficiency received the routine iv HC; 3 pts got 100 mg in the morning before surgery, and 2 got $100 \mathrm{mg}$ in the morning plus $50 \mathrm{mg}$ during surgery 8 hours after the first dose as they had surgery in the afternoon (Fig. 2). 